\title{
STUDY OF THE DYNAMICAL MODEL OF HIV
}

\author{
M.A. Lapshova, E.A. Shchepakina \\ Samara National Research University, Samara, Russia
}

\begin{abstract}
The paper is devoted to the study of the dynamical model of HIV. An application of the technique of singular perturbation theory allows us to calculate the conditions for the stabilization of the HIV status of the patient depending on the features that reflect the different nature of the intervention of doctors in the treatment process.
\end{abstract}

Keywords: HIV, predator-prey model, stability.

Citation: Lapshova MA, Shchepakina EA. Study of the dynamical model of HIV. CEUR Workshop Proceedings, 2016; 1638: 600-609. DOI: 10.18287/1613-0073-2016-1638-600-609

\section{Introduction}

There are many diseases that cause death nowadays. One of the most widespread disease is human immunodeficiency virus (HIV). HIV is slowly progressive viral infection of the immune system that causes weakening of the immune defense against infections. Acquired immune deficiency syndrome (AIDS) is the last stage of HIV. Concentration of immune cells so low at that stage, that the body can't fight the virus. About 60 million people have been infected HIV and 25 million people have died of AIDS since the beginning of epidemic. Therefore research of this infection is very important.

Studying of the mathematical model allows us to determine the dynamics of the virus and the body's immune response depending on the function that describes medical intervention.

\section{Model}

The following system of ordinary differential equations describes the dynamic of HIV infection [1]:

$$
\begin{aligned}
\dot{x}(t) & =\alpha-\lambda x v-d x-f(y, v) x, \\
\dot{y}(t) & =\lambda x v-a y, \\
\dot{v}(t) & =k y-u v, \\
\dot{z}(t) & =\mu v-b z,
\end{aligned}
$$


here $x(t)$ is the concentration of healthy CD4+T immune cells, $y(t)$ is the concentration of infected CD4+T cells, $v(t)$ is the concentration of free virus particles, and $z(t)$ is the concentration of antigen-specific CTL. Parameter $\alpha$ is a rate of generating of healthy CD4+T cells, $d$ is a rate of dying, free virus particles infect the healthy cell at a rate $\lambda, a$ is a death rate of infected cells, the rates of producing and removing of free virus particles are described by indexes $k$ and $u$, respectively. The specific CTL proliferate at a rate $\mu$ and decay at a rate $b$. All arguments are nonnegative. Function $f$ describes protection of immune cells against penetration of viral particles and may depends on concentration of infected CD4+T cells and on concentration of free virus particles. According to behavior of concavity of this function the dynamics of system solution are different. The research of principal cases is our goal.

\section{Analysis}

The system (1)-(4) is multiscale in time. The equation (3) is a fast stage (cycle corresponds to 12 hours), equation (4) is the fastest stage (cycle corresponds to 3-4 hours). For this reason we introduce the new small positive parameters $\varepsilon_{1}$ and $\varepsilon_{2}$ to transform system (1)-(4) into singularly perturbed form:

$$
\begin{aligned}
& \dot{x}(t)=\alpha-\lambda x v-d x-f(y, v) x, \\
& \dot{y}(t)=\lambda x v-a y, \\
& \varepsilon_{1} \dot{v}(t)=k y-u v, \\
& \varepsilon_{1} \varepsilon_{2} \dot{z}(t)=\mu v-b z .
\end{aligned}
$$

Typically, to investigate a singularly perturbed system a combination of asymptotic and geometrical techniques of analysis are applied [2,3]. The essence of this approach consists in separating out the slow motions of the system under investigation. Then the order of the differential system decreases, but the reduced system, of a lesser order, inherits the essential elements of the qualitative behaviour of the original system in the corresponding domain and reflect the behaviour of the original models to a high order of accuracy when the slow integral manifold is attracting. A mathematical justification of this method can be given by means of the theory of integral manifolds for singularly perturbed systems [3].

System (5)-(6) is a slow subsystem, and (7)-(8) is a fast subsystem of the full system. Setting $\varepsilon_{1}=0$ and $\varepsilon_{2}=0$ we obtain the degenerate system:

$$
\begin{aligned}
& \dot{x}(t)=\alpha-\lambda x v-d x-f(y, v) x, \\
& \dot{y}(t)=\lambda x v-a y, \\
& k y-u v=0, \\
& \mu v-b z=0 .
\end{aligned}
$$


The equations (11)-(12) describe the so-called slow surface of system (5)-(8) [2, 3]. The slow surface is the zero order approximation (i. e., as $\varepsilon_{1} \rightarrow 0$ and $\varepsilon_{2} \rightarrow 0$ ) of the slow integral manifold. The slow integral manifold is defined as an invariant surface of slow motions (see [3] and references therein).

The Jacobian matrix of (11)-(12)

$\left(\begin{array}{cc}-u & 0 \\ \mu & -b\end{array}\right)$

has the eigenvalues

$\lambda_{1}=-u, \quad \lambda_{2}=-b$.

Note that $\lambda_{1}$ and $\lambda_{2}$ are negative $(u>0$ and $b>0)$, hence the slow surface is attractive. For the reason above $[4,5]$ we can reduce the system with help (9), (10), and instead of system (5)-(8) we will analyze its projection on the zero order approximation of the slow integral manifold:

$\dot{x}(t)=\alpha-\beta x y-d x-f(y, v) x, \dot{y}(t)=\beta x y-a y$,

where $\beta=k \lambda / u$ is the extent of infection.

The following cases are considered in the present paper:

- $f(y)=y^{2}$,

- $f(y)=\frac{c y}{1+\gamma y}$,

- $\quad f(y)=\frac{c y^{2}}{1+\gamma y}$.

It should be noted that we have $f(y)=0$ if $\mathrm{y}=0$ for all cases (14)-(16).

The equilibrium points of system (13) are determined by the following system:

$\{\alpha-\beta x y-d x-f(y) x=0$,

$$
\beta x y-a y=0 .
$$

The equilibrium point $(\alpha / d, 0)$ corresponds to the case without infected cells. The Jacobian matrix of (13) at this point is

$$
\left(\begin{array}{cc}
-d & -\beta \frac{\alpha}{d} \\
0 & \beta \frac{\alpha}{d}-a
\end{array}\right) .
$$

The characteristic equation

$$
(\lambda+d)\left(\lambda-\beta \frac{\alpha}{d}+a\right)=0
$$

has the roots

$\lambda_{1}=-d, \lambda_{2}=\beta \frac{\alpha}{d}-a$. 
Note that $\lambda_{1}$ is always negative. Hence, the equilibrium point $(\alpha / d, 0)$ is a sink if

$\beta \frac{\alpha}{d}-a<0$

and, in other way, it is a saddle. For example, values of arguments: $\beta=0.216, a=0.8$, $\alpha=0.1, d=0.06$ satisfy the inequality (17) with the interior equilibrium at $(1.67,0)$ And in this case the concentration of healthy cells increases and stabilizes, see Fig. 1.
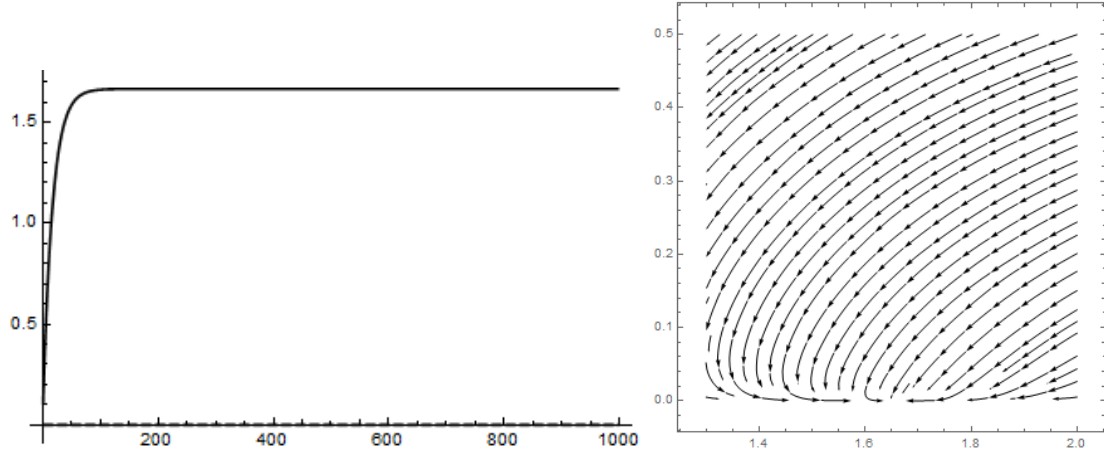

Fig. 1. (left) Concentration of healthy cells and (right) the phase portrait for system (13) with

$$
\beta=0.216, a=0.8, \alpha=0.1, d=0.06
$$

For the case (14) system (13) takes the form

$\left\{\begin{array}{l}\dot{x}(t)=\alpha-\beta x y-d x-y^{2} x, \\ \dot{y}(t)=\beta x y-a y .\end{array}\right.$

The equilibrium point with coordinates $x=x^{*}, y=y^{*}$, where

$$
x^{*}=\frac{a}{\beta}, y^{*}=\frac{-a \beta \pm \sqrt{-4 a^{2} d+4 a \alpha \beta+a^{2} \beta^{2}}}{2 a} \text {, }
$$

corresponds to the case of the infected cells' presence. Recall that the variables $x$ and $y$ are positive, then system (18) has an unique positive steady state

$\left(\frac{a}{\beta}, \frac{-a \beta+\psi}{2 a}\right), \psi=\sqrt{-4 a^{2} d+4 a \alpha \beta+a^{2} \beta^{2}}$.

Moreover, the variable $y$ must be real-valued, thus we get constraint:

$-4 a^{2} d+4 a \alpha \beta+a^{2} \beta^{2} \geq 0$

from which we get $\alpha \beta>a d$. 
The characteristic equation at the critical point $(a / \beta,(-a \beta+\psi) /(2 a))$ is

$$
\lambda^{2}+\frac{\alpha \beta \lambda}{a}-2 a d+2 \alpha \beta+0.5 a \beta^{2}-0.5 \beta \psi=0 .
$$

If the discriminant

$$
D=\left(\frac{\alpha \beta}{a}\right)^{2}-4\left(-2 a d+2 \alpha \beta+0.5 a \beta^{2}-0.5 \beta \psi\right)
$$

of equation (19) is more than zero then the first root

$$
\lambda_{1}=-\frac{1}{2}\left(\frac{\alpha \beta}{a}+\sqrt{8 a d-8 \alpha \beta-2 a \beta^{2}+\frac{\alpha^{2} \beta^{2}}{a^{2}}+2 \beta \psi}\right)
$$

is negative. Hence if the second root

$$
\lambda_{2}=-\frac{1}{2}\left(\frac{\alpha \beta}{a}-\sqrt{8 a d-8 \alpha \beta-2 a \beta^{2}+\frac{\alpha^{2} \beta^{2}}{a^{2}}+2 \beta \psi}\right)
$$

is positive then the critical point $(a / \beta,(-a \beta+\psi) /(2 a))$ is a sink otherwise it is a saddle. For example, for parameters' values $\beta=3, a=0.3, \alpha=0.2, d=0.02$ the critical point is a sink and has the coordinates $(0.100,0.557)$. Figure 2 shows the solutions' plots and the phase portrait of system (18) in the neighborhood of the critical point for this case. From the graphs one can see that the concentrations of healthy (the solid curve) and infected (the dashed curve) cells increase at first, but then the number of infected cells continues to grow while the number of healthy cells is reduced. Although the status of patient is stabilized but the number of infected cells more than the number of normal cells. Thus, this case corresponds to the severe disease.

If the discriminant (20) is equal to zero then

$$
\lambda_{1}=\lambda_{2}=-\frac{\alpha \beta}{2 a}<0
$$

and the critical point is a stable degenerate node. If the discriminant (20) is less than zero, we have

$$
\lambda_{1,2}=-\frac{1}{2}\left(\frac{\alpha \beta}{a} \pm i \sqrt{8 a d-8 \alpha \beta-2 a \beta^{2}+\frac{\alpha^{2} \beta^{2}}{a^{2}}+2 \beta \psi}\right)
$$

and the point $(a / \beta,(-a \beta+\psi) /(2 a))$ is a stable focus. For example, for parameters' values $\beta=3, a=0.8, \alpha=0.2, d=0.02$ the condition $D>0$ is fulfilled and the critical 
point has the coordinates $(0.267,0.226)$. The graphs of the solutions and the phase portrait in the neighborhood of the critical point $(0.267,0.226)$ are presented in Figure 3 . Under these conditions, concentration of healthy cells (the solid curve) and infected cells (the dashed curve) are damped oscillations at the beginning, followed by stabilization. The number of healthy cells exceeds the number of infected cells after the initial oscillations.
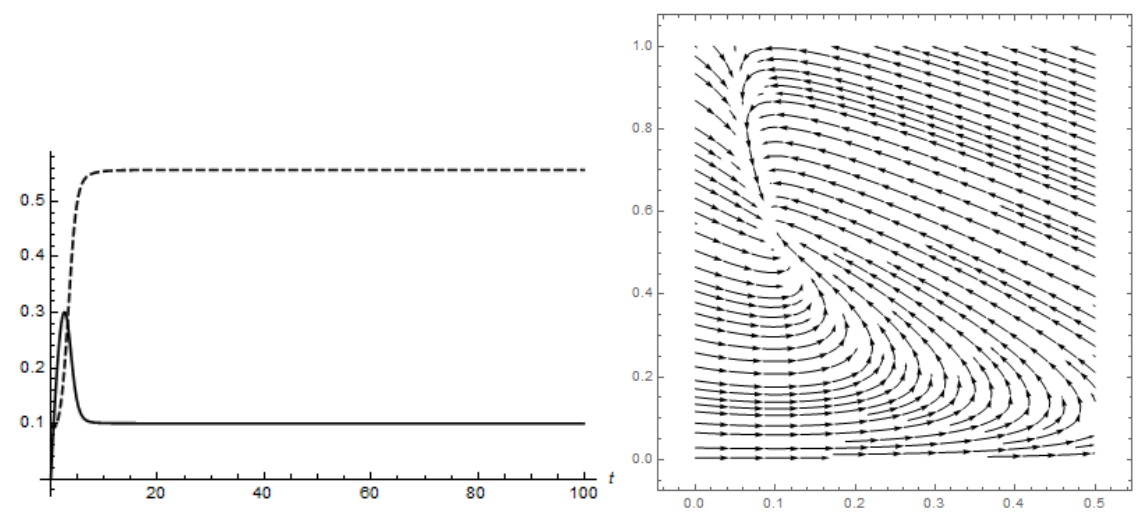

Fig. 2. (left) Concentration of healthy (the solid curve) and infected (the dashed curve) cells and (right) the phase portrait for system (18) with $\beta=3, a=0.3, \alpha=0.2, d=0.02$

In the case (15) system (13) takes the form

$\left\{\begin{array}{l}\dot{x}(t)=\alpha-\beta x y-d x-\frac{c y}{1+\gamma y} x, \\ \dot{y}(t)=\beta x y-a y .\end{array}\right.$
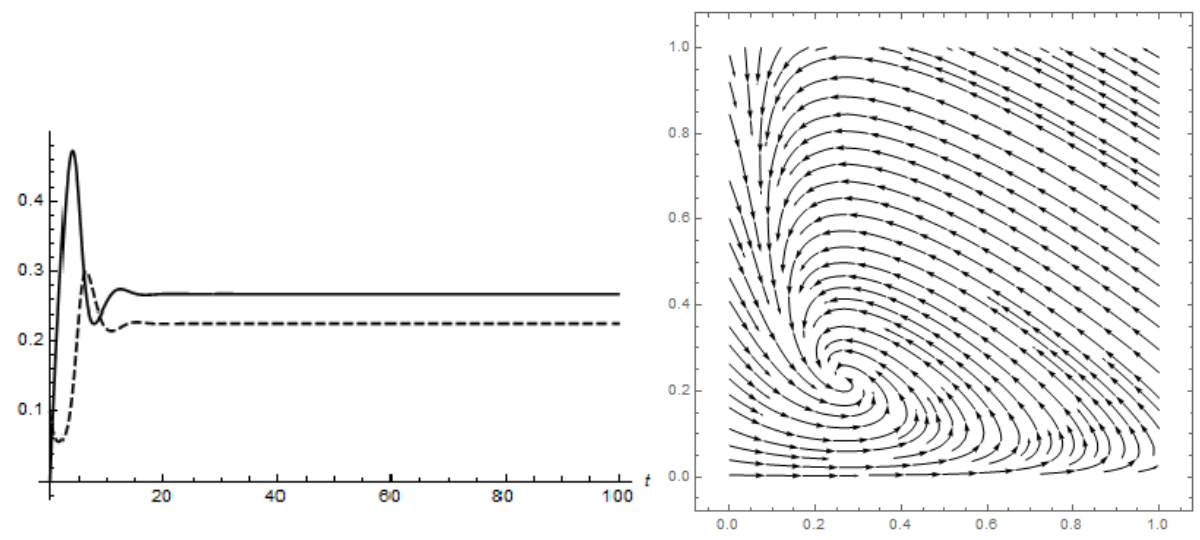

Fig. 3. (left) Concentration of healthy (the solid curve) and infected (the dashed curve) cells and (right) the phase portrait for system (18) with $\beta=3, a=0.8, \alpha=0.2, d=0.02$ 
The positive equilibrium state with coordinates

$$
\begin{aligned}
& \left(\frac{a}{\beta}, \frac{1}{2 a \beta \gamma}(-a c-a \beta-a d \gamma+\alpha \beta \gamma+\xi)\right), \\
& \xi=\sqrt{-4 a \beta(a d-\alpha \beta) \gamma+(a c+a \beta+a d \gamma-\alpha \beta \gamma)^{2}},
\end{aligned}
$$

corresponds to the case of presence of the infected cells. Note that the number of the cells concentrations must be real under condition

$(a c+a \beta+a d \gamma-\alpha \beta \gamma)^{2}>4 a \beta(a d-\alpha \beta) \gamma$.

The characteristic equation at this critical point is

$$
\begin{aligned}
\lambda^{2}+ & \lambda\left(\frac{d}{2}+\frac{\alpha \beta}{2 a}-\frac{c+\beta}{2 \gamma}+\frac{\xi}{2 a \gamma}+\frac{c \alpha}{a \omega}-\frac{c d}{\beta \omega}-\frac{c}{\gamma \omega}-\frac{c^{2}}{\beta \gamma \omega}+\frac{c \xi}{a \beta \gamma \omega}\right)-\frac{a d-\alpha \beta}{2} \\
& -\frac{a c+a \beta-\xi}{2 \gamma}+\frac{c \alpha}{2(1+\gamma)^{2}}-\frac{a c d}{2 \beta(1+\gamma)^{2}}-\frac{a c}{2 \gamma(1+\gamma)^{2}}-\frac{a c^{2}-c \xi}{2 \beta \gamma(1+\gamma)^{2}}=0,
\end{aligned}
$$

where

$$
\omega=2\left(1+\frac{\alpha \beta \gamma+\xi-a c-a \beta-a d \gamma}{2 a \beta}\right) .
$$

Let us set

$$
\begin{aligned}
& a_{1}=\frac{d}{2}+\frac{\alpha \beta}{2 a}-\frac{c+\beta}{2 \gamma}+\frac{\xi}{2 a \gamma}+\frac{c \alpha}{a \omega}-\frac{c d}{\beta \omega}-\frac{c}{\gamma \omega}-\frac{c^{2}}{\beta \gamma \omega}+\frac{c \xi}{a \beta \gamma \omega}, \\
& a_{2}=\frac{1}{2}\left(-a d+\alpha \beta-\frac{a c+a \beta-\xi}{\gamma}+\frac{c \alpha}{(1+\gamma)^{2}}-\frac{a c d}{\beta(1+\gamma)^{2}}-\frac{a c}{\gamma(1+\gamma)^{2}}-\frac{a c^{2}-c \xi}{\beta \gamma(1+\gamma)^{2}}\right) .
\end{aligned}
$$

According to the Routh Hurwitz stability criterion the system is stable at a critical point if we have

$$
\Delta_{1}=a_{1}>0
$$

and

$$
\Delta_{2}=\left|\begin{array}{cc}
a_{1} & 1 \\
0 & a_{2}
\end{array}\right|>0 .
$$

Due to $\Delta_{2}=a_{2} \Delta_{1}$ the second inequality implies $a_{2}>0$. The graphics of the solutions and the phase portrait in the neighborhood of this critical point are plotted for 
values of the parameters $\beta=0.216, a=0.4, \alpha=0.2, d=0.02, c=0.1, \gamma=2$, and are presented in Figure 4. Note that for these values of the parameters the critical point has the coordinates $(1.852,0.317)$ and the stability conditions are fulfilled. The figure shows that the concentration of healthy cells and infected cells are stabilized at the same time after several periods of damped oscillations. The number of healthy cells prevails over the number of infected cells. In this case a patient continues to live and feels well.
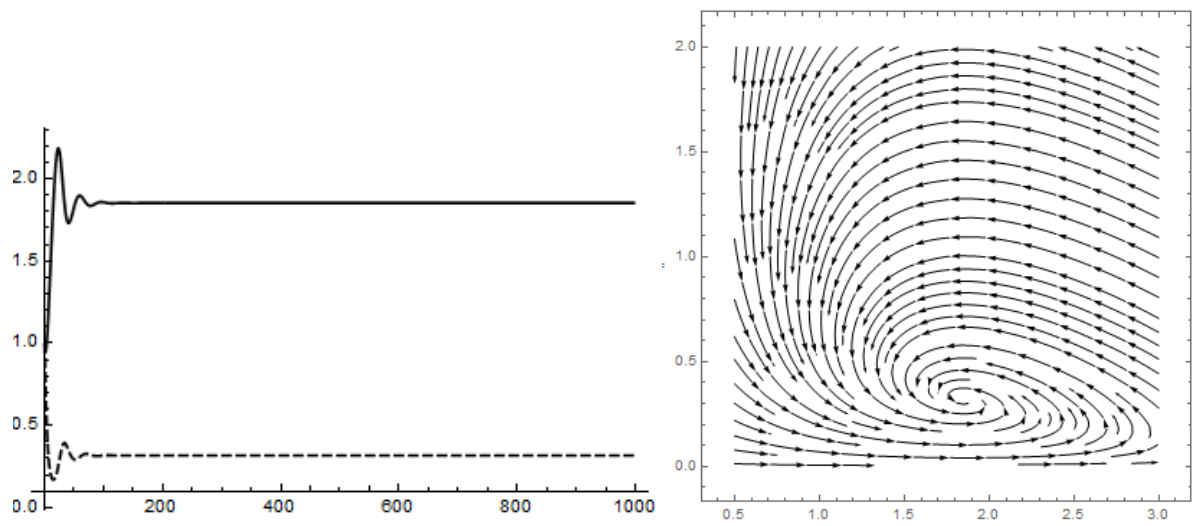

Fig. 4. (left) Concentration of healthy (the solid curve) and infected (the dashed curve) cells and (right) the phase portrait for system (21) with

$$
\beta=0.216, a=0.4, \alpha=0.2, d=0.02, c=0.1, \gamma=2
$$

In the case (16) system (13) has the form

$$
\left\{\begin{array}{l}
\dot{x}(t)=\alpha-\beta x y-d x-\frac{c y^{2}}{1+\gamma y} x, \\
\dot{y}(t)=\beta x y-a y .
\end{array}\right.
$$

The positive equilibrium state with coordinates

$$
\left(\frac{a}{\beta}, \frac{-a \beta-a d \gamma+\alpha \beta \gamma+\varphi}{2(a c+a \beta \gamma)}\right),
$$

where

$$
\varphi=\sqrt{-4(a d-\alpha \beta)(a c+a \beta \gamma)+(a \beta+a d \gamma-\alpha \beta \gamma)^{2}}
$$

corresponds to the case of presence of infected cells. For real values of variable $y$ we need

$$
(a \beta+a d \gamma-\alpha \beta \gamma)^{2}>4(a d-\alpha \beta)(a c+a \beta \gamma) .
$$


The stabilization conditions are found like in the previous case and are

$$
\begin{aligned}
\left(2 a^{2} c^{2} d\right. & -a^{2} c^{2} \beta-a^{2} c \beta^{2}-a^{2} c^{2} d \gamma+a c^{2} \alpha \beta \gamma-a^{2} c \beta^{2} \gamma+3 a c \alpha \beta^{2} \gamma \\
& -a^{2} c d^{2} \gamma^{2}-a^{2} c d \beta \gamma^{2}+a c d \alpha \beta \gamma^{2}+a c \alpha \beta^{2} \gamma^{2}+a \alpha \beta^{3} \gamma^{2} \\
& -a d \alpha \beta^{2} \gamma^{3}+\alpha^{2} \beta^{3} \gamma^{3}+a c^{2} \phi+a c \beta \phi+a c d \gamma \phi+a c \beta \gamma \phi \\
& \left.+\alpha \beta^{2} \gamma^{2} \phi\right) /\left(a(c+\beta \gamma)\left(2 a c+a \beta \gamma-a d \gamma^{2}+\alpha \beta \gamma^{2}+\gamma \phi\right)\right)>0
\end{aligned}
$$

and

$$
\begin{aligned}
&-\left(\left(2 \left(a^{3}\left(4 c^{2} d+d \gamma^{2}(\beta-d \gamma)^{2}-c\left(\beta^{2}-2 d \beta \gamma+5 d^{2} \gamma^{2}\right)\right)\right.\right.\right. \\
&- \alpha^{2} \beta^{2} \gamma^{3}(\alpha \beta \gamma+\vartheta-a \alpha \beta \gamma(c(5 \alpha \beta \gamma+3 \vartheta+\gamma(\alpha \beta \gamma(2 \beta-3 d \gamma) \\
&+(\beta-2 d \gamma) \vartheta+a^{2}\left(-4 c^{2} \alpha \beta-\gamma^{2}(-\beta+d \gamma)(-\alpha \beta(\beta-3 d \gamma)\right. \\
&+d \vartheta+c(-2 \alpha \beta \gamma(\beta-5 d \gamma)+(\beta+3 d \gamma) \vartheta))) \\
& \times\left(2 a c+a \beta \gamma-a d \gamma^{2}+\alpha \beta \gamma^{2}+\gamma \phi\right)^{-1}>0 .
\end{aligned}
$$

Here $\vartheta=\sqrt{\alpha^{2} \beta^{2} \gamma^{2}+2 a \alpha \beta(2 c+\gamma(\beta-d \gamma))+a^{2}\left(-4 c d+(\beta-d \gamma)^{2}\right)}$.

For example, for

$\beta=0.216, a=0.4, \alpha=0.2, d=0.02, c=0.1, \gamma=2$

the critical point has the coordinates $(1.852,0.371)$ and the plots of the solutions and the phase portrait in the neighborhood of the critical point are shown at Figure 5. The critical point is asymptotically stable.
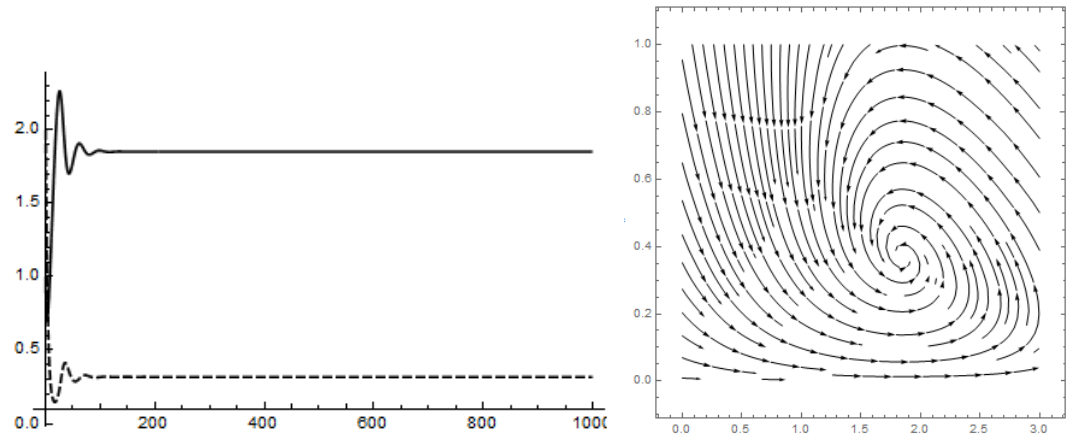

Fig. 5. (left) Concentration of healthy (the solid curve) and infected (the dashed curve) cells and (right) the phase portrait for system (22) with

$$
\beta=0.216, a=0.4, \alpha=0.2, d=0.02, c=0.1, \gamma=2
$$




\section{Conclusion}

Thus, in the cases considered above the status of a patient infected with the virus may be stabilized under certain conditions which could be achieved by selection of the appropriate treatment. It should be noted that the application of the geometric theory of the singular perturbation made it possible to solve several actual problems concerning multirate processes in biological systems, see, for example, [6-9].

\section{Acknowledgements}

This work is supported in part by the Russian Foundation for Basic Research (grant 14-01-97018-p) and the Ministry of Education and Science of the Russian Federation under the Competitiveness Enhancement Program of Samara University (2013-2020).

\section{References}

1. Huang G, Takeuchi Y, Korobeinikov A. HIV evolution and progression of the infection to AIDS. J. Theor. Biol., 2012; 307: 149-159.

2. Shchepakina E, Sobolev V. Black swans and canards in laser and combustion models. Chapter 8 (51 p.) in "Singular Perturbations and Hysteresis", ed. by Mortell MP, O’Malley R, Pokrovskii A, Sobolev V. Philadelphia: SIAM, 2005; 344 p.

3. Shchepakina E, Sobolev V, Mortell MP. Singular Perturbations: Introduction to System Order Reduction Methods with Applications. In: Springer Lecture Notes in Mathematics, Vol. 2114. Cham: Springer International Publishing, 2014; 212 p.

4. Kononenko LI, Sobolev VA. Asymptotic expansion of slow integral manifolds. Sib. Math. J., 1994; 35: 1119-1132.

5. Korobeinikov A, Shchepakina E, Sobolev V. Paradox of enrichment and system order reduction: bacteriophages dynamics as case study. Mathematical Medicine and Biology, July, 2015. URL: http://www.ncbi.nlm.nih.gov/pubmed/26180103.

6. Archibasov AA, Korobeinikov A, Sobolev VA. Order reduction for an RNA virus evolution model. Journal Mathematical Biosciences and Engineering, 2015; 12(5): 1007-1016.

7. Archibasov AA, Korobeinikov A, Sobolev VA. Asymptotic expansions for solutions of singularly perturbed model of viral evolution. Journal of Computational Mathematics and Mathematical Physics, 2015; 2: 242-252. [in Russian]

8. Sobolev VA. Canards and the effect of apparent disappearance. CEUR Workshop Proceedings, 2015; 1490: 190-197. DOI: 10.18287/1613- 0073-2015-1490-190-197.

9. Gavin C, Pokrovskii A, Prentice M, Sobolev V. Dynamics of a Lotka-Volterra type model with applications to marine phage population dynamics. Journal of Physics: Conference Series, 2006; 55: 80-93. 